2001

\section{March 2001}

9th Annual Public Health Forum People Policies, Effective Practice

Date: 27.03.01-29.03.01

Venue: Bournemouth

Contact: Profile Productions Ltd,

Northumberland House, 11 The Pavement, Popes Lane, London, W5 4NG.

Tel: +44 (0)20 88327300

Fax: +44(0)20 88327301

Annual Meeting of the Craniofacial Society of Great Britain - Craniofacial Management in the 21st Century

Date: 28.03.01 - 30.03.01

Venue: Trinity College, Dublin, Ireland Contact: Congress Secretariat, Craniofacial Society of Great Britain and Ireland, Abbey Conference Services, City Gates, 22 Bridge Street, Lr Dublin 8, Ireland.

Tel: +35316799144

Fax: +35316798194

E-mail: abbey@abbey.ie.

\section{April 2001}

British Society for the Study of Prosthetic Dentistry 48th Annual Conference

Date: 8.04.01 - 10.04.01

Venue: Britannia International Hotel, London Contact: Mr R. D. Welfare, Dept of Prosthetic Dentistry, Eastman Dental Institute, 256 Gray's Inn Road, London. WC1X 8LD. Tel: $+44(0) 2079151073$

Fax: +44 (0)207915 1246

E-mail: r.welfare@eastman.ucl.ac.uk

Dental Practice Board Conference

Date: 23.04.01 - 24.04.01

Venue: Cumberland Hotel, London

Contact: Ms Heather Deacon, Room D19,

Dental Practice Board, Compton Place

Road, Eastbourne, East Sussex. BN20 8AD.

Tel: +44(0)1323433307

Fax: +44 (0)1323 433517

E-mail: h.deacon@dpb.nhs.uk

Oral \& Maxillofacial Non Consultant Career Grade National Conference
Date: $24.04 .01-27.04 .01$

Venue: Liverpool

Contact: Mrs Rosemary Thomas,

Southport \& Ormskirk District General

Hospital, Town Lane, Southport, Merseyside PR8 6PN.

Tel: +44 (0) 1704704085

Annual Irish Scientific Conference 2001

Date: 25.04.01 - 28.04.01

Venue: Kilkenny Ormonde Hotel,

Kilkenny, Ireland

Contact: Irish Dental Association,

10 Richview Office Park, Clonskeagh

Road, Dublin 14.

Tel: 0035312830499

Fax: 0035312830515

\section{May 2001}

BDA Conference 2001: Best Practice

Date: 03.05 .01 - 05.05.01

Venue: Harrogate International Centre

Contact: Ms Charlotte Long, Events Office,

64 Wimpole Street, London W1M 8AL

Tel: +44 (0)20 79350875 ext 286

Fax: +44 (0)20 74860855

E-mail: c.long@bda-dentistry.org.uk

30th Australian Dental Congress

Date: 04.05 .01 - 08.05.01

Venue: Brisbane Convention and Exhibi-

tion Centre, Australia

Contact: Jacki Mole, Event Manager,

Congress Secretariat, P.O. Box 1280

11/97 Castlemaine Street, Milton,

Queensland 4064, Australia

Tel: +61 733690477

Fax: +61 733691512

E-mail:ada2001@im.com.au

The British Society for Oral Medicine Annual Meeting

Date: 10.5 .01 - 11.05.01

Venue: University of Birmingham Conference Park, Birmingham

Contact: Mr John Hamburger, Unit of

Oral Medicine, University of Birmingham,

School of Dentistry, St Chad's Queensway,

Birmingham $\mathrm{B} 46 \mathrm{NN}$.

Tel: $+44(0) 1212372888$
Fax: +44 (0)121625 8815

E-mail: J.Hamburger@bham.ac.uk

British Society for Restorative Dentistry Spring Scientific Meeting

Date: $11.05 .01-12.05 .01$

Venue: Cedar Court Hotel, Harrogate Contact: Ms Janet Harlow, British Society for Restorative Dentistry, Turner Dental School, Higher Cambridge Street, Manchester, M15 6FH.

Tel: +44 (0)161 2756797

Fax: +44 (0)1612756797

E-mail: janet.Harlow@man.ac.uk

Joint meeting of the British Society of Periodontology, the International Academy of Periodontology and the British Dental Hygienists' Association: Periodontics in Practice

Date: 17.05 .01 - 19.05.01

Venue: Glasgow Royal Concert Hall

Contact: Mrs Ann Hallowes, Administra-

tive Secretary, British Society of Periodontology, 44 Pool Road, Hartley, Hook. RG27 8RD.

Tel: $+44(0) 1252843598$

Fax: +44 (0) 1252844018

E-mail: bspadmin@btinternet.com

\section{September 2001}

FDI World Dental Congress 2001

Date: 27.09.01-01.10.01

Venue: Kuala Lumpur

Contact: FDI, 7 Carlisle Street,

London W1V 5RG

Tel: +44 (0)2079357852

Fax: +44 (0)2074860183

Website: www.fdi.org.uk

British Orthodontic Conference

Date: 30.09.01 - 03.10.01

Venue: Harrogate International Centre

Contact: Mrs Ann Wright, British Orthodontic Conference, BOS Office, 291 Gray's Inn Road, London WC1X 8QF.

Tel: +44 (0)20 78372193

Fax: +44 (0)20 78372193

E-mail: awrightbos@msn.com 